\title{
Src family kinase-mediated vesicle trafficking is critical for neutrophil basement membrane penetration
}

\section{Ina Rohwedder, ${ }^{1}$ Angela R.M. Kurz, ${ }^{1}$ Monika Pruenster, ${ }^{1}$ Roland Immler, ${ }^{1}$ Robert Pick, ${ }^{1}$ Tanja Eggersmann, ${ }^{1}$ Sarah Klapproth, ${ }^{1}$ Jennifer L. Johnson, ${ }^{2}$ Sergi Masgrau Alsina, ${ }^{1}$ Clifford A. Lowell, ${ }^{3}$ Attila Mócsai, ${ }^{4}$ Sergio D. Catz ${ }^{2}$ and Markus Sperandio ${ }^{1}$}

${ }^{1}$ Walter-Brendel-Center of Experimental Medicine, Institute of Cardiovascular Physiology and Pathophysiology, Klinikum der Universität, Ludwig-Maximilians-University Munich, Planegg-Martinsried, Germany; ${ }^{2}$ Department of Molecular Medicine, The Scripps Research Institute, La Jolla, CA, USA; ${ }^{3}$ Department of Laboratory Medicine, University of California, San Francisco, CA, USA and ${ }^{4}$ Department of Physiology, Semmelweis University School of Medicine, Budapest, Hungary

\section{ABSTRACT}

L eukocyte recruitment into inflamed tissue is highly dependent on the activation and binding of integrins to their respective ligands, followed by the induction of various signaling events within the cell referred to as outside-in signaling. Src family kinases (SFK) are the central players in the outside-in signaling process, assigning them a critical role for proper immune cell function. Our study investigated the role of SFK on neutrophil recruitment in vivo using $\mathrm{Hck}^{-} \mathrm{Fgr}^{-1} \mathrm{Lyn}^{-}$mice, which lack SFK expressed in neutrophils. We show that loss of SFK strongly reduces neutrophil adhesion and post-arrest modifications in a shear force dependent manner. Additionally, we found that in the absence of SFK, neutrophils display impaired Rab27adependent surface mobilization of neutrophil elastase, VLA3 and VLA6 containing vesicles. This results in a defect in neutrophil vascular basement membrane penetration and thus strongly impaired extravasation. Taken together, we demonstrate that SFK play a role in neutrophil post-arrest modifications and extravasation during acute inflammation. These findings may support the current efforts to use SFK-inhibitors in inflammatory diseases with unwanted neutrophil recruitment.

\section{Introduction}

Chronic inflammatory diseases are an increasing problem in western industrialized countries. A hallmark of these disorders is the recruitment of leukocytes from the circulation into affected tissues. This process follows a well-defined cascade of activation and adhesion events starting with the initial capture of leukocytes from the blood stream, followed by rolling along inflamed endothelium. ${ }^{1}$ Both steps are mediated by selectins interacting with glycosylated ligands on leukocytes. ${ }^{2}$ Through binding of the leukocyte specific integrin LFA1 ( $\alpha \mathrm{L} \beta 2)$ to ICAM-1 on endothelial cells, leukocytes slow down their rolling velocity, and in combination with chemokine stimulation, firmly arrest on the endothelial surface. This is followed by post-arrest modifications, a process characterized by cell spreading, cytoskeleton rearrangements and crawling along the endothelium, that is critical for tight adhesion to the substrate and allows an appropriate spot for extravasation into tissue. Transmigration involves the crossing of the venular wall and the underlying vascular basement membrane (BM), two steps which are still incompletely understood. Several reports suggest a role for the integrins VLA3 ( $\alpha 3 \beta 1)$ and VLA6 $(\alpha 6 \beta 1)$ along with neutrophil elastase (NE) in this process. ${ }^{3.5}$ Recently, our group has shown that neutrophils translocate VLA3, VLA6 and NE from internally stored vesicles to the cell surface, to subsequently cross the vascular BM. ${ }^{6}$ The release of these vesicles is initiated by interactions of neutrophils with the inflamed endothelium in a PECAM-1/ICAM-1- and CXCL1-dependent manner. Vesicle transport is
Haematologica 2020

Volume 105(7):1845-1856

\section{Correspondence:}

MARKUS SPERANDIO

markus.sperandio@Imu.de

Received: April 29, 2019.

Accepted: November 5, 2019.

Pre-published: November 7, 2019.

doi:10.3324/haematol.2019.225722

Check the online version for the most updated information on this article, online supplements, and information on authorship \& disclosures: www.haematologica.org/content/105/7/1845

\section{(C)2020 Ferrata Storti Foundation}

Material published in Haematologica is covered by copyright. All rights are reserved to the Ferrata Storti Foundation. Use of published material is allowed under the following terms and conditions:

https://creativecommons.org/licenses/by-nc/4.0/legalcode. Copies of published material are allowed for personal or internal use. Sharing published material for non-commercial purposes is subject to the following conditions: https://creativecommons. org//icenses/by-nc/4.0/legalcode, sect. 3. Reproducing and sharing published material for commercial purposes is not allowed without permission in writing from the publisher. 
mainly regulated by Rab GTPases and their effector proteins with Rab27a being the main Rab molecule involved in the secretory machinery of neutrophils. ${ }^{7}$ Rab27a function is mediated by the two effector molecules synaptotagmin-like protein 1 (JFC1, encoded by Syt11 in mice) and Munc13-4 (Unc13d). ${ }^{8}$ Although most of these processes rely on integrin signaling, integrins themselves lack enzymatic activity. Therefore, numerous proteins are recruited to their intracellular tails, among those Src family kinases (SFK). Their early recruitment during integrin activation assigns SFK a critical role in the so-called outside-in signaling process and thus regulation of central signaling pathways downstream of integrin-receptor ligation. ${ }^{9}$ Neutrophils express three members of this family, namely Hck, Fgr and Lyn. ${ }^{10}$ Their function has been intensively studied in SFK single, double ( $\left.\mathrm{Hck}^{-F} \mathrm{Fr}^{-\prime}\right)$, and triple knockout (ko) $\left(H_{c k}{ }^{-1} g^{-1} L y n^{-/}\right)$mice, ${ }^{11}$ demonstrating a role for SFK in integrin activation ${ }^{12}$ and their downstream signaling. Neutrophils isolated from $\mathrm{Hck}^{\wedge} \mathrm{Fgr}^{\curvearrowright}$ mice showed poor spreading, indicating that integrin outside-in signaling is impaired..$^{13}$ Additionally, neutrophil migration into the liver of $\mathrm{Hck}^{-} \mathrm{Fgr}^{-}$mice in an in vivo endotoxemia model is severely reduced. ${ }^{14}$ Furthermore, Kovács et al. recently showed that neutrophils exhibit reduced capability to create a microinflammatory environment, resulting in diminished neutrophil extravasation in a model of autoantibody-induced arthritis and inflammatory blistering skin disease..$^{15}$

Our study aimed to investigate the effect of SFK on leukocyte recruitment in an in vivo setting of acute inflammation, focusing on post-arrest modifications and the molecular mechanism of vascular BM penetration. We show that, in the genetic absence of SFK, these two steps are strongly impaired. Adherent $\mathrm{Hck}^{\prime} \mathrm{Fgr} / \mathrm{Lyn}^{\prime}$ neutrophils are unable to withstand shear forces and display diminished LFA1 clustering with reduced phosphorylation levels of Paxillin, Cortactin and Syk, suggesting that SFK depletion results in severely impaired adhesion strengthening. In addition, we show that SFK are critical for crossing the vascular BM during neutrophil extravasation by facilitating translocation of VLA3-, VLA6- and NE-containing vesicles to the cell surface. Therefore, SFK are not only important mediators of neutrophil post-arrest modifications, but are also required to breach the vascular BM.

\section{Methods}

\section{Animals}

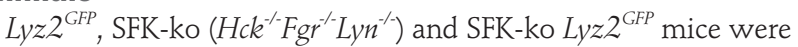
generated as described earlier. ${ }^{16-18}$ C57BL/6 wildtype mice were purchased from Janvier Labs (Saint Berthevin, France). All animal experiments were approved by the Regierung von Oberbayern, Germany (AZ 55.2-1-54-2531-80-76/12 and 55.2-1-54-2532-1022017).

\section{Live cell imaging of in vitro laminin digestion}

Transmigration of neutrophils was analyzed in $\mu$-Slide membrane ibiPore flow chambers (Ibidi, Planegg, Germany) equipped with a 300-nm thick membrane with $5-\mu \mathrm{m}$ pores, and a subjacent rat-tail collagen gel $(1.5 \mathrm{mg} / \mathrm{mL})$ containing $10 \mu \mathrm{M} \mathrm{N}$-formylmethionyl-leucyl-phenylalanine (fMLP) as chemoattractant. The upper compartment was coated with Laminin (LN), PECAM-1 and ICAM-1, and, in addition, LN was visualized using an anti-LN antibody conjugated to Alexa Fluor-647 (novusbio, Littleton, CO,
USA). Isolated neutrophils from wildtype and SFK-ko animals were labeled with CellTracker ${ }^{\mathrm{TM}}$ Green CMFDA Dye and CellTracker ${ }^{\mathrm{TM}}$ Red CMTPX Dye (Thermo Fisher, Waltham, MA, USA), respectively, and distributed into the upper chamber compartment in a 1:1 ratio. Time-lapse microscopy with an interval of 50 seconds was performed using an upright spinning-disk confocal microscope (Examiner; Zeiss) equipped with a confocal scanner unit CSU-X1 (Yokogawa Electric Corporation, Japan), an EMCCD camera (Evolve; Photometrics), and a x20/1.0 NA water immersion objective (Plan Apochromat; Zeiss). 3D images (70 z-stacks with a step size of $2 \mu \mathrm{m}$ ) were acquired per time point and analyzed by generating maximum z-projections over time using Slidebook 6.0.8 software (3i) and ImageJ. Interaction strength was analyzed with the MosaicIA interaction plugin of Fiji.

Functional in vitro and in vivo experiments, including intravital microscopy, ${ }^{19}$ peritonitis experiments, flow chamber experiments, vesicle trafficking, flow cytometry, western blot analysis and statistics are described in detail in the Online Supplementary Methods.

\section{Data sharing statement}

Original data are available on request.

\section{Results}

\section{Src family kinase depletion reduces neutrophil adhesion and extravasation in inflamed postcapillary venules in vivo}

We first investigated how loss of neutrophil-expressed SFK influences neutrophil adhesion in TNF $\alpha$-stimulated cremaster muscle postcapillary venules using intravital microscopy. Interestingly, the absolute number of adherent neutrophils was strongly increased compared to wildtype control mice (Online Supplementary Figure S1A). However, after adjusting the number of adherent neutrophils to the circulating neutrophil count, which was significantly higher in SFK-ko mice compared to wildtype mice (Online Supplementary Figure $S 1 B$ and C), the neutrophil adhesion efficiency calculated as number of adherent cells $/ \mathrm{mm}^{2}$ divided by the systemic neutrophil count was significantly reduced in SFK-ko mice $(0.48 \pm 0.04) \mathrm{com}$ pared to wildtype mice $(1.04 \pm 0.08)$ (Figure 1A). This was not due to altered surface expression of rolling and adhesion relevant surface proteins including CD18, CD11a, CD11b, CD62L, PSGL1, CXCR2, and CD44 as their surface expression was equal between wildtype and SFK-ko neutrophils (Online Supplementary Figure S1D). We therefore conclude that SFK are critical for neutrophil adhesion in vivo.

Efficient neutrophil extravasation into tissue is required for proper host defense. Recently, Kovács et al. demonstrated in the $\mathrm{K} / \mathrm{B} \times \mathrm{N}$ serum-transfer arthritis model that SFK deficiency resulted in lower levels of cytokine production and release leading to impaired leukocyte extravasation..$^{15}$ To investigate a cell intrinsic adhesion defect in SFK-ko mice, we injected TNF $\alpha$ into the mouse scrotum. This approach circumvents possible effects of a reduced inflammatory environment and enables us to focus on neutrophil recruitment itself. Performing Giemsa staining of TNF $\alpha$ stimulated cremaster muscle whole mounts revealed strongly reduced numbers of extravasated neutrophils in SFK-ko mice compared to wildtype mice (306.51 \pm 43.7 vs. $578.6 \pm 72.9$, respectively) (Figure $1 \mathrm{~B}$ and Online Supplementary Figure S1E). Moreover, we calculated the extravasation efficiency as total number of extravasat- 
ed leukocytes divided by the total number of adherent leukocytes to differentiate between reduced extravasation due to decreased adhesion versus a specific extravasation defect. Surprisingly, we observed a strongly reduced extravasation efficiency in SFK-ko mice (0.21 in SFK-ko mice vs. 0.77 in wildtype mice) (Figure 1C), indicating that SFK depletion does not only affect neutrophil adhesion, but also interferes with extravasation itself. Finally, we also compared neutrophil cell numbers in the peritoneal cavity 2 hours (h) after TNF $\alpha$-induced peritonitis (Figure 1D). In SFK-ko animals, peritoneal neutrophil numbers were reduced to $50 \%$ compared to wildtype mice $\left(0.42 \times 10^{6}\right.$ vs. $10^{6}$, respectively). Taken together, these experiments demonstrate an important role for SFK in neutrophil extravasation.

\section{Adhesion strengthening is severely impaired in Src} family kinase-knockout neutrophils in vitro

Our experiments suggest that SFK-deletion in neutrophils results in an extravasation defect in the presence of the proinflammatory cytokine TNF $\alpha$. After firm adhesion to the inflamed endothelium, neutrophils start to polarize and crawl along the endothelium to find a suitable spot for extravasation. These changes are highly dependent on $\beta 2$ integrins LFA1 $(\alpha L \beta 2)$ and Mac1 $(\alpha M \beta 2)$ and integrin outside-in signaling. ${ }^{20}$ We analyzed neutrophil crawling using time-lapse microscopy in TNF $\alpha$ stimulated cremaster muscle venules of SFK-ko and wildtype mice. We observed no differences in crawling direction (Figure 2A), but, interestingly, crawling velocity was significantly increased in SFK-ko mice $(12.3 \mu \mathrm{m} / \mathrm{min}) \mathrm{com}$ pared to wildtype mice $(10.7 \mu \mathrm{m} / \mathrm{min})$ (Figure $2 \mathrm{~B})$, suggesting defective adhesion strengthening. Subsequently, we performed ex vivo flow chamber assays using glass capillaries coated with E-Selectin/ICAM-1/CXCL1 to mimic the inflamed endothelium. Plots of single cell tracks displayed more SFK-ko neutrophils crawling in flow direction compared to wildtype neutrophils (Figure 2C). Crawling velocity was increased in SFK-ko leukocytes (Figure 2D), verifying our in vivo results. Interestingly, only $20 \%$ of SFK-ko neutrophils were able to crawl under flow (Figure 2E and Online Supplementary Mov1), compared to $80 \%$ of wildtype cells, suggesting that SFK-ko neutrophils are unable to maintain stable adhesion to the substrate. Together, these findings demonstrate a decreased capability of SFK-ko neutrophils to withstand shear forces, indicating that SFK are necessary for adhesion strengthening during post-arrest modifications. To further investigate a shear stress dependent adhesion defect in the absence of neutrophil SFK, we conducted detachment assays in the
A

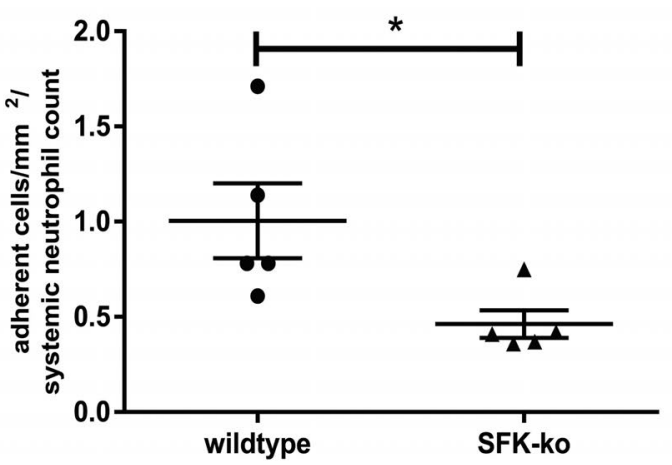

C

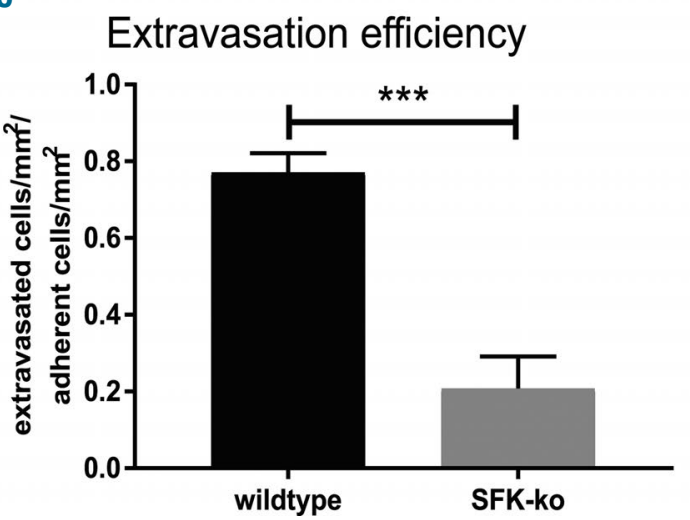

B

Extravasation

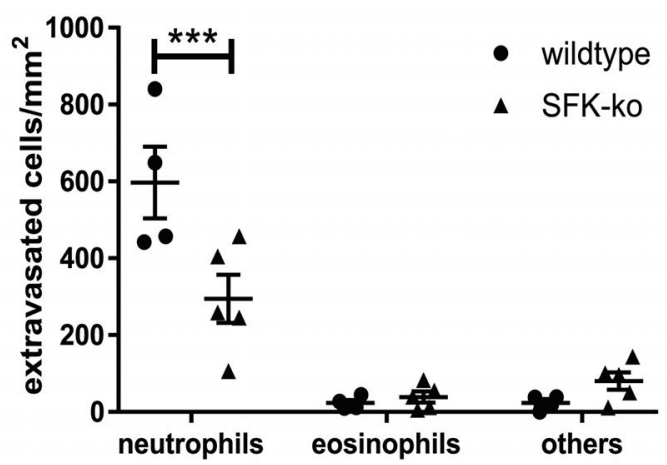

D

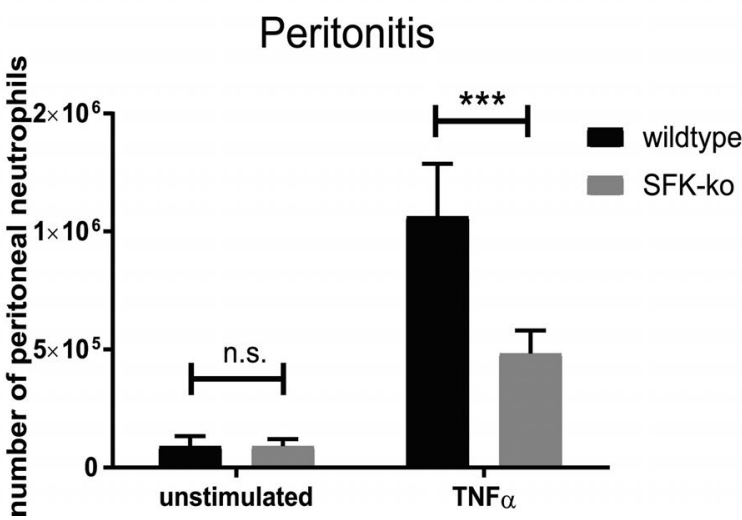

Figure 1. Src family kinases (SFK) are required for slow rolling, neutrophil adhesion and extravasation. All data are presented as mean \pm standard error of the mean (SEM). $* P<0.05 ; * * * P<0.001$; n.s. : not significant (unpaired Student $t$-test or two-way ANOVA, Sidak multiple comparison test). (A) Adhesion efficiency from wildtype or SFK-knockout (ko) mice: $n=5$ wildtype; $n=5$ SFK-ko mice. (B) In vivo leukocyte extravasation in TNF $\alpha$-stimulated venules of mouse cremaster muscle. Differential total cell counts of neutrophils, eosinophils and other cells: $n=4$ wildtype; $n=5$ SFK-ko mice. (C) Extravasation efficiency was calculated by the number of extravasated cells $/ \mathrm{mm}^{2}$ divided by number of adherent cells $/ \mathrm{mm}^{2}$. (D) Total neutrophil numbers after peritoneal lavage of the unstimulated or TNF $\alpha$-stimulated peritoneal cavity of wildtype and SFK-ko mice: $n=7$ wildtype and $n=6$ SFK-ko mice for unstimulated controls and $n=6$ wild-type and $n=6$ SFK-ko mice for TNF $\alpha$. 
flow chamber using increasing shear forces. Adherent neutrophils were assessed for each shear stress level and related to the number of adherent neutrophils under low flow conditions (Figure 2F). Loss of SFK resulted in a decrease in shear stress resistant adhesion compared to wildtype cells. At wall shear stress levels of $140 \mathrm{dyn} / \mathrm{cm}^{2}$, only $36 \%$ of SFK-ko leukocytes could adhere to the flow chamber, while $84 \%$ of wildtype leukocytes were still attached. To analyze if these findings also apply under in vivo conditions, we correlated in vivo shear rates to
A

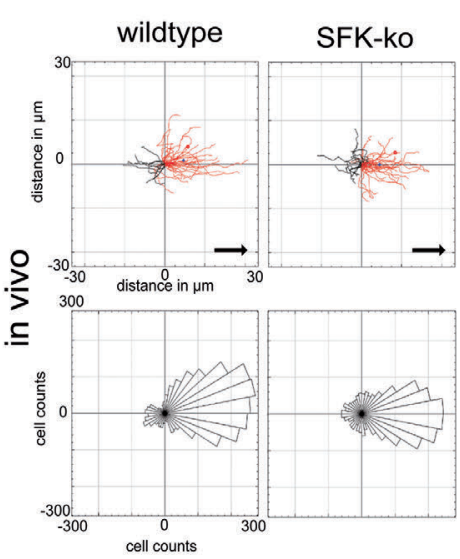

C

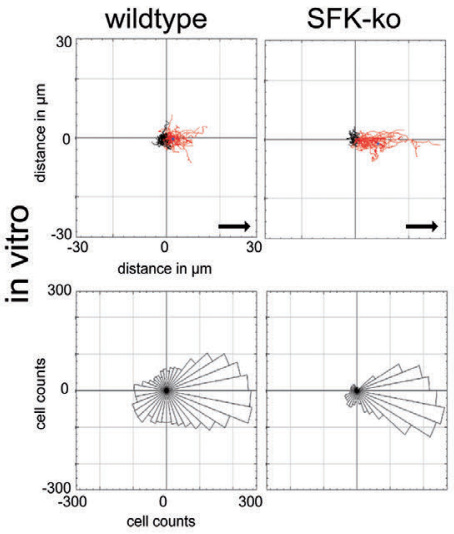

E

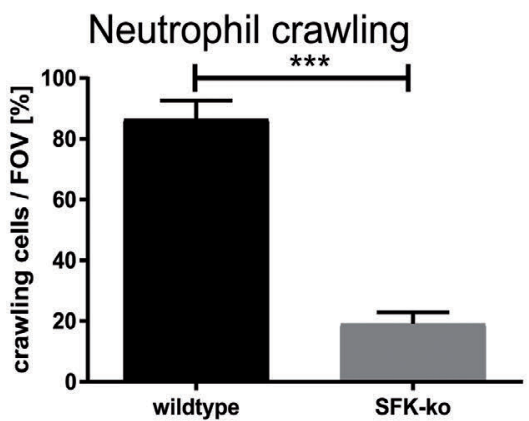

G

wildtype

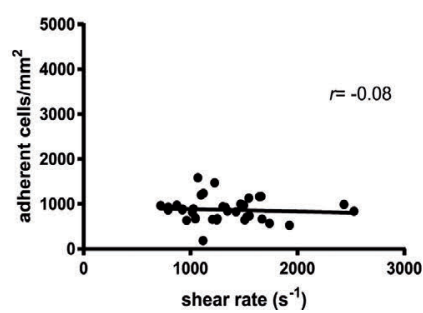

B

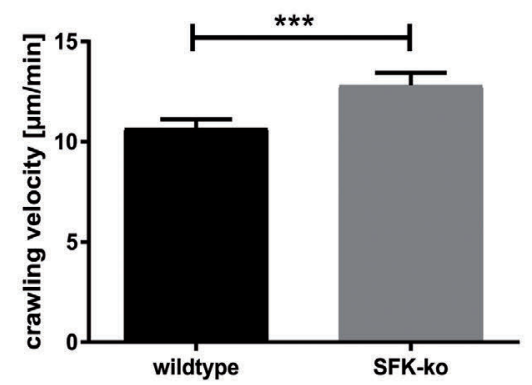

D

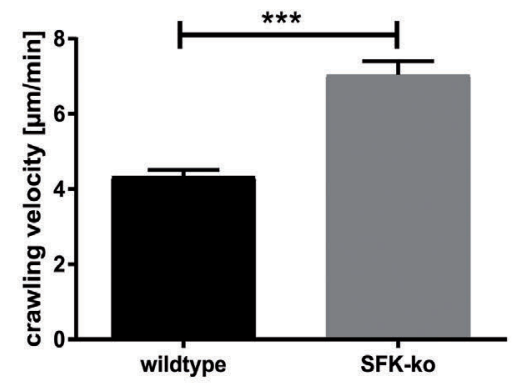

$\mathbf{F}$

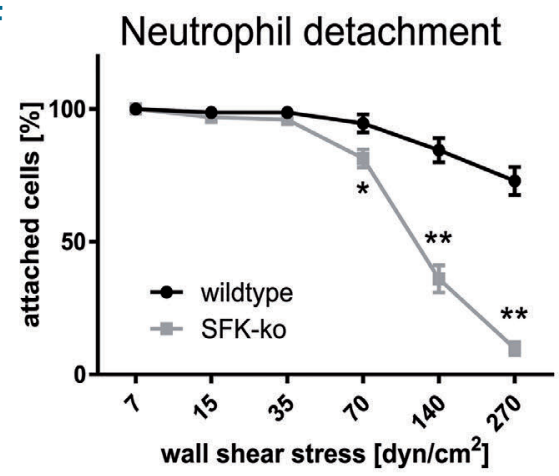

Figure 2. Neutrophil shear stress resistance is impaired in the genetic absence of Src family kinases (SFK). In vivo crawling experiments were analyzed in TNF $\alpha$-stimulated cremaster muscle tissue of 5 wildtype and 5 SFKknockout (ko) mice. Time-lapse movies over 15 minutes were performed and leukocytes were tracked for $15 \mathrm{~min}$ utes. In vitro crawling experiments were analyzed using whole blood from 3 wildtype and 3 SFK-ko mice in flow chambers coated with ESelectin/ICAM-1 and CXCL1. Timelapse movies over 10 minutes were performed and leukocytes tracked for 200 time points. All data are presented as mean \pm standard error of the mean (SEM). $* P<0.05 ; * * P<0.005$ $* * * P<0.001$; n.s. : not significant (unpaired Student $t$-test). (A and C) Representative single cell migration tracks (upper panel) and rose plots (lower panel) for crawling in vivo (A) and in vitro $(\mathrm{C})$ are displayed. Red lines indicate migration in, black lines migration against flow direction. Arrows indicate the direction of flow. In rose diagrams, the area of each sector is proportional to the frequency of the migration vectors of tracked wildtype or SFK-ko neutrophils. At least 50 cells were analyzed for each strain. (B) Intraluminal crawling velocity. (D) Crawling velocity under flow in vitro. $(E)$ Relative number of crawling cells per Field of View (FOV) in vitro using wild type and SFK-ko neutrophils. (F) Neutrophil detachment was analyzed using whole blood from 4 wildtype and 4 SFK-ko mice in flow chambers coated with E-Selectin/ICAM-1 and CXCL1. Flow was applied and increased every 30 seconds as indicated and numbers of attached cells per FOV counted. All data are presented as mean \pm SEM. $* P<0.05 ; * * P<0.005 ; * * * \quad P<0.001$ (two-way ANOVA, Sidak multiple comparison test). (G) In vivo correlation of shear rates and number of adherent cells in TNF $\alpha$-stimulated cremaster muscle venules of 5 wildtype and 5 SFK-ko mice and 5 wildtype mice treated for 3 hours with the tyrosine kinase inhibitor Dasatinib (10 mg/kg).
SFK-ko

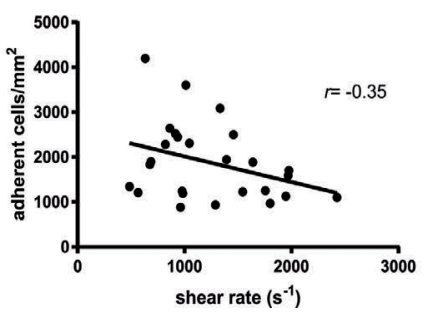

wildtype + Dasatinib $(10 \mathrm{mg} / \mathrm{kg})$

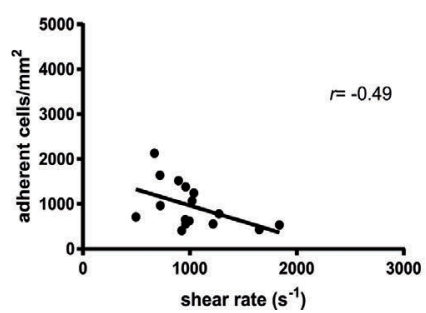


absolute numbers of adherent cells $/ \mathrm{mm}^{2}$ in TNF $\alpha$-stimulated post-capillary cremaster muscle venules of SFK-ko and wildtype mice (Figure 2G). We found a similar shear rate dependent decrease in the number of adherent cells $/ \mathrm{mm}^{2}$ in SFK-ko mice in vivo compared to wildtype mice. Additionally, we used a pharmacological approach and administered the broad-spectrum tyrosine kinase inhibitor Dasatinib before TNF $\alpha$-stimulation. Dasatinib has been used in various studies as a SFK inhibitor and led to promising results as an immune modulator. ${ }^{21}$ Similar to the results in SFK-ko mice, we found a decrease in adherent cells with increasing shear rates. Importantly, SKF-ko mice receiving Dasatinib did not show any further decrease in adherent cells at high shear stress levels, indicating that Dasatinib exerts its observed effects on adhesion via inhibition of SFK (Online Supplementary Figure $S 1 F)$. Taken together, these in vivo and in vitro findings indicate that loss of SFK in neutrophils leads to a shear force dependent inability to firmly adhere to the inflamed endothelium, a prerequisite for subsequent extravasation.

\section{Reduced phosphorylation of cytoskeleton-associated proteins in Src family kinase-deficient neutrophils}

To further elucidate the molecular mechanisms of SFK dependent neutrophil extravasation, we analyzed different steps of integrin outside-in signaling that occur after integrins bind to their receptor. This includes integrin clustering and cytoskeletal rearrangements. ${ }^{22}$ To analyze integrin clustering, we performed time-lapse confocal microscopy and studied LFA1 clustering in SFK-ko and wildtype neutrophils in flow chambers coated with E-selectin/ICAM-1/CXCL1 (Figure 3A and Online Supplementary Mov2). We observed spreading and polarizing wild-type neutrophils which showed an accumulating LFA1 signal (clustering) over time at the neutrophil uropod (Figure 3B). In contrast, SFK-deficient neutrophils appeared round and unpolarized with no obvious LFA1 clustering. Several cytoskeleton-associated proteins and signaling molecules are known to be tyrosine phosphorylated upon integrin ligation. ${ }^{23} \mathrm{We}$ investigated the phosphorylation and activation of the direct SFK target ${ }^{24,25}$ Syk in SFK-ko and wildtype neutrophils plated on ICAM-1 and then stimulated with CXCL1 or PMA. Western blot analysis of phospho-Syk (Tyr519/520) revealed a strong upregulation of phosphorylation following stimulation of wildtype cells. In contrast, SFK deficiency prevented the upregulation of phosphorylation following stimulation with CXCL1 or PMA (Figure 3C), indicating that Syk activation is defective in the absence of SFK. Moreover, we tested the phosphorylation of the adaptor protein Paxillin, one of the critical proteins for cell adhesion, migration and podosome formation, that is known to be tyrosine phosphorylated upon $\beta_{2}$ integrin activation. ${ }^{26,27}$ Western blot analysis showed significant upregulation of Paxillin phosphorylation when stimulated with CXCL1 or PMA in wildtype cells, while no upregulation was detectable in SFK-ko neutrophils (Figure 3D). In addition, we also investigated the tyrosine phosphorylation of Cortactin, which regulates actin branching and therefore cell migration. Likewise, we observed no significant upregulation of Cortactin Tyr421 phosphorylation upon CXCL1 or PMA stimulation in SFK-ko cell, in contrast to wildtype neutrophils (Figure 3E). Because during neutrophil adhesion and migration SFK do not selectively signal via LFA1, but also via Mac1, we additionally analyzed Paxillin phospho- rylation after plating the cells on Fibrinogen (Online Supplementary Figure S1G). Similar to ICAM1 we observed a stimulus dependent phosphorylation of Paxillin in wildtype neutrophils, which was absent in SFK-ko neutrophils. Taken together, these experiments show that $\beta_{2}$-integrin clustering and subsequent outside-in signaling is severely impaired in SFK-ko neutrophils.

\section{Src family kinase are indispensable to pass the vascular basement membrane}

For successful extravasation into inflamed tissue, neutrophils need to cross the endothelial cell layer and the underlying vascular basement membrane. We aimed to investigate whether SFK are required to perform this last step of extravasation. First, we analyzed transmigration capacity of SFK-ko neutrophils in a transwell assay using CXCL1 as chemoattractant (Online Supplementary Figure $S 2 A)$. In line with previous results, ${ }^{15}$ we observed no difference in transmigration between SFK-ko neutrophils and wildtype neutrophils. Both groups displayed a proportional increase in transmigration with rising CXCL1 concentrations. As a next step, we coated transwells with laminin-111 (LN1) or a combination of LN1 and PECAM1/ICAM-1 and stimulated them with CXCL1 in order to mimic the situation at the vascular BM, as reported previously. ${ }^{6}$ No difference in transmigration through LN alone was detected between wildtype and SFK-ko neutrophils (Figure 4A). Coating with LN1/PECAM-1/ICAM-1 (LN/P/I) together with CXCL1 stimulation induced a strong increase in transmigration of wildtype cells. However, SFK-ko cells failed to cross the artificial BM. How exactly neutrophils cross the vascular BM is still a matter of debate, especially, whether the BM is permanently modified by extravasating neutrophils. To further address this, we conducted transwell assays as described above, coated with a combination of LN1 and PECAM1/ICAM-1 and stimulated with CXCL1. We also used the same total cell number $\left(2 \times 10^{5}\right)$, but this time mixed SFKko $x$ Lyz2GFP neutrophils and wildtype neutrophils in a 1:1 ratio $\left(1 \times 10^{5}\right.$ wildtype and $1 \times 10^{5}$ SFK-ko $x$ Lyz2 GFP neutrophils). In this manner, transmigrated SFK-ko neutrophils were identified by their GFP signal. Interestingly, in combination with wildtype neutrophils, SFK-ko neutrophils were able to overcome the BM to the same extent as wildtype cells, suggesting that wildtype neutrophils facilitate BM penetration by providing exit points for SFKko neutrophils (Figure 4A). To confirm these findings in vivo, we performed whole mount staining of TNF $\alpha$ stimulated cremaster muscles for LN (green) to visualize the BM, and MRP14 (red) to visualize neutrophils, then analyzed the localization of neutrophils by confocal microscopy. In wildtype tissue, we were able to detect local areas of increased extravasation, which is in accordance with published literature, describing transmigration hotspots $^{28}$ (Figure 4B). In contrast, none of these spots could be found in cremaster muscle tissue of SFK-ko mice. MPR14-positive cells remained located within the vascular compartment and only few extravasated neutrophils could be detected, mainly remaining in close vicinity to the vessel (white arrows). Overall, SFK-ko neutrophils covered less distance away from the vessel wall when compared to wildtype neutrophils $(8.0 \mu \mathrm{m}$ vs. $17.3 \mu \mathrm{m}$, respectively) (Online Supplementary Figure S2B). These in vivo findings support our earlier in vitro findings that SFK are critical for neutrophils to cross the BM. 

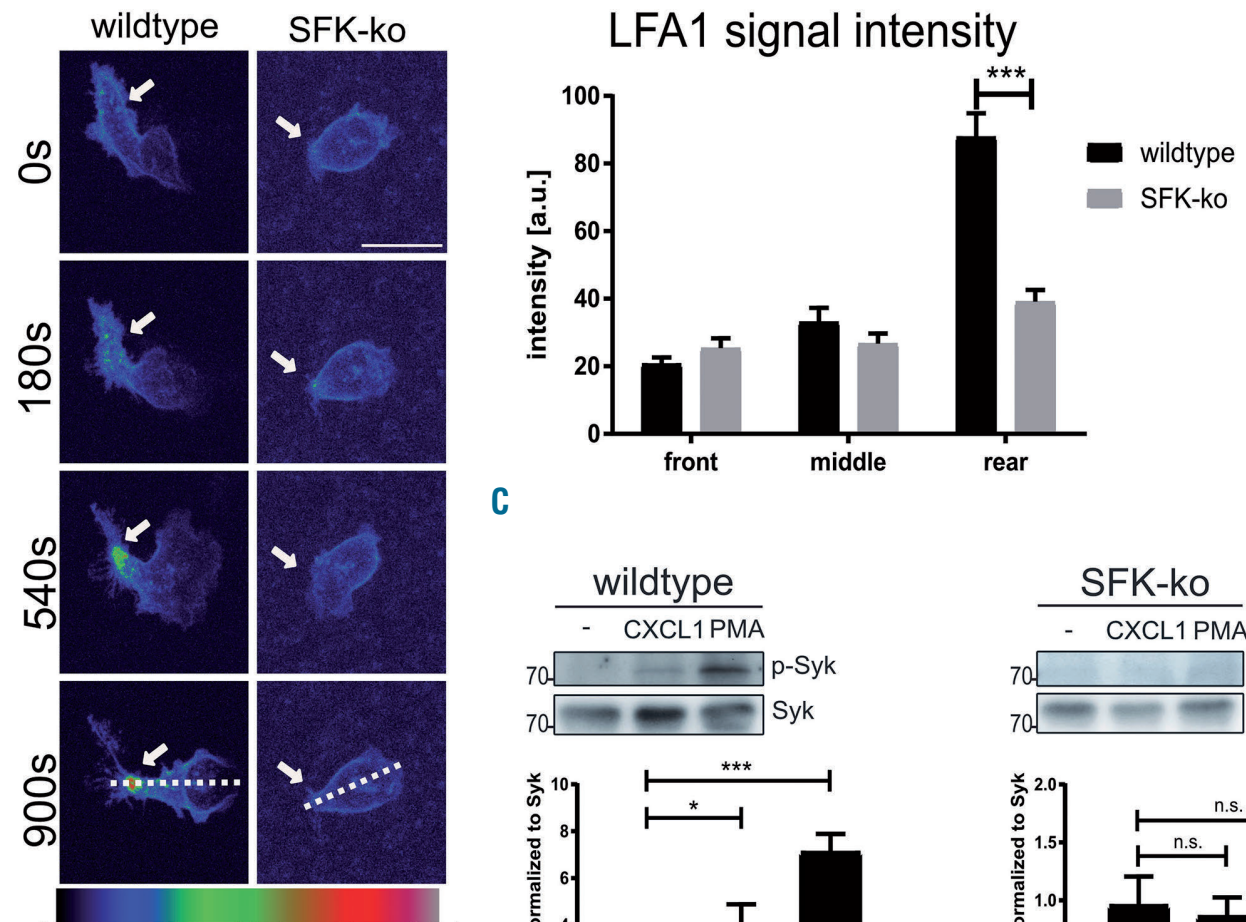

C
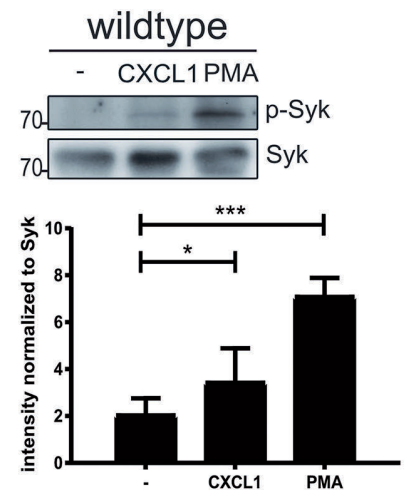

D
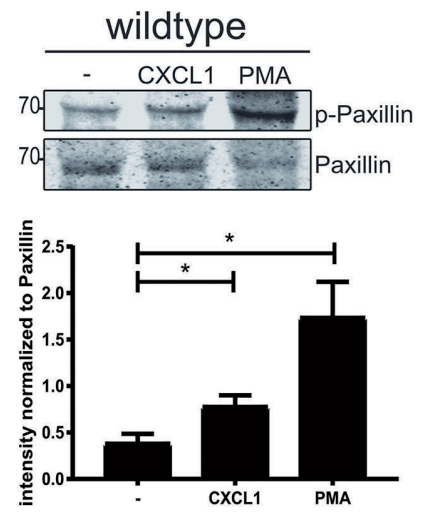

E
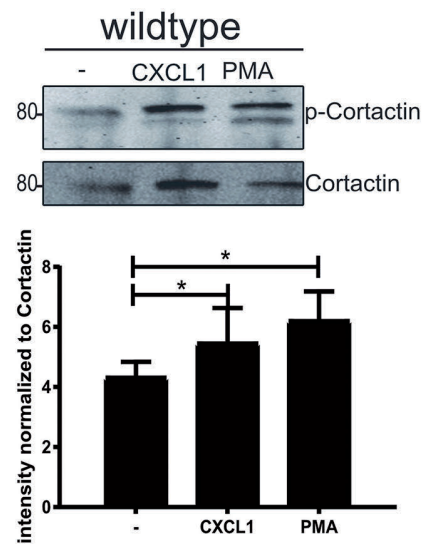
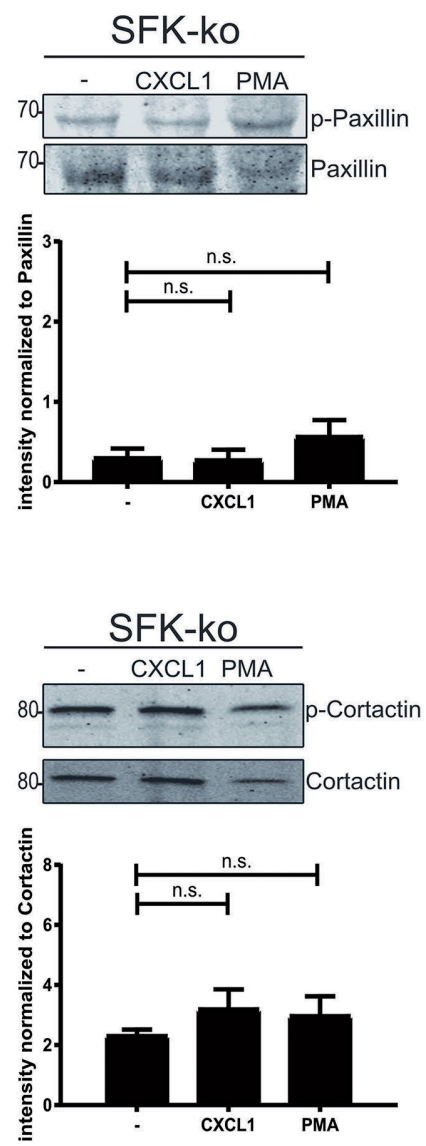

Figure 3. Defective LFA1 clustering and adhesion strengthening in Src family kinases (SFK)-knockout (ko) mice. Integrin downstream signaling was analyzed in isolated wildtype and SFK-knockout (ko) neutrophils. (A) In vitro integrin clustering experiments were performed using whole blood from wildtype and SFK-ko mice in flow chambers coated with E-Selectin/ICAM-1 and CXCL1. Blood samples were incubated with anti-LFA1-Alexa547 (2D7) to visualize LFA1 and crawling cells were analyzed for 15 minutes by confocal microscopy. LFA1 intensity is displayed at different time points. White arrows point to the uropod. Color code indicates signal intensity. Dashed lines are exemplary for drawn intensity profiles analyzed in (B) $n=3$ wildtype and 3 SFK-ko mice. Scale bar: 5 um. (B) Mean signal intensities of an intensity profile of LFA1 signal, grouped into 3 regions: front, middle and rear of the neutrophil. (C-E) Western blot and respective quantitative analysis of Syk (Tyr519/520) (72kDa), Paxillin (Tyr118) (68kDa) and Cortactin (Tyr421) (80kDa) phosphorylation after CXCL1 and PMA stimulation in wildtype and SFK-ko neutrophils plated on ICAM1. Band intensity was normalized to respective total protein. All data are presented as mean \pm standard error of the mean (SEM). $* P<0.05$; $* * * P<0.001$; n.s. : not significant (one-way ANOVA, Dunnett's multiple comparisons test). $\mathrm{N}=3$ wildtype and 3 SFK-ko mice. 
A

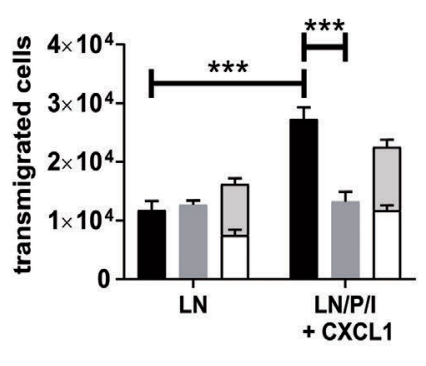

B

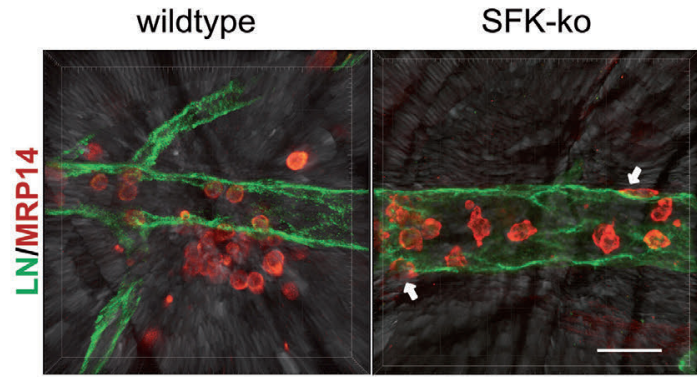

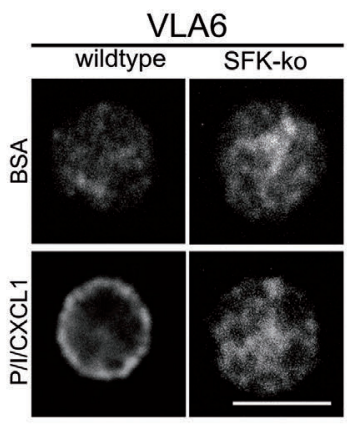

D
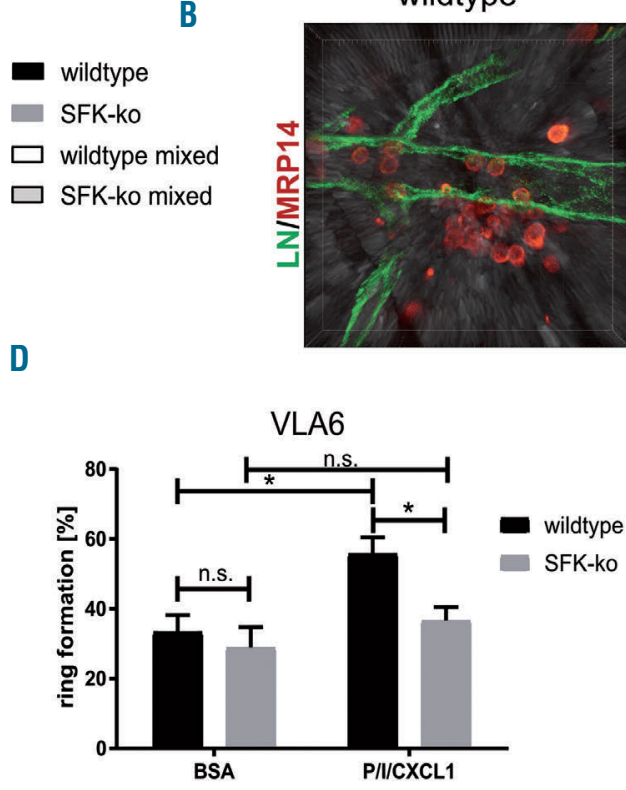

E

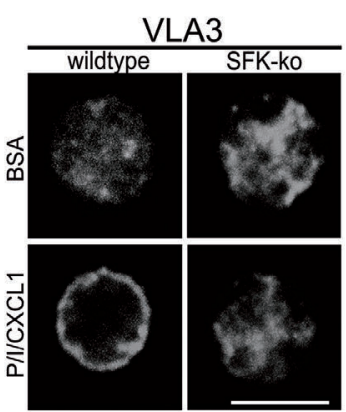

F

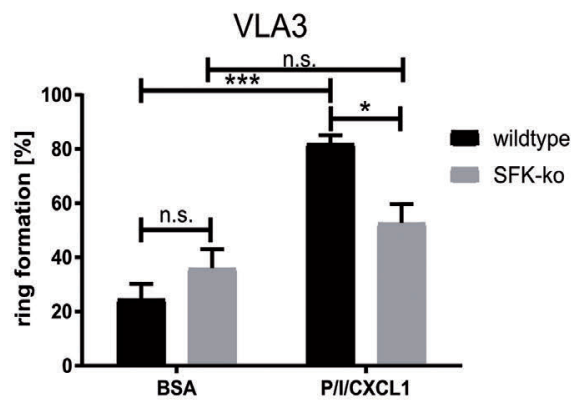

G

wildtype
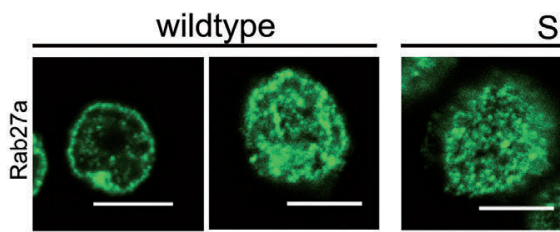

SFK-ko

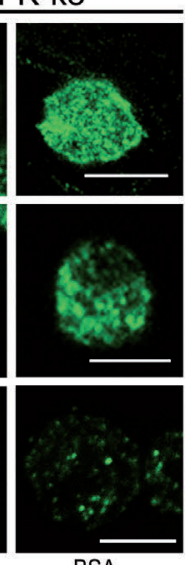

H
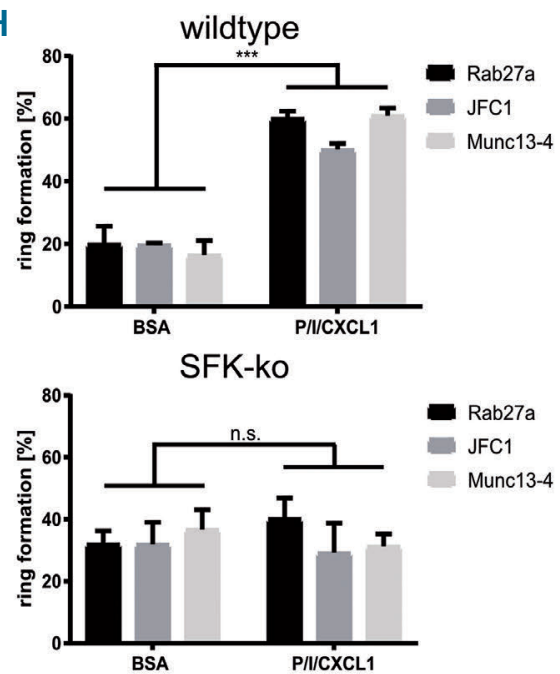

- Rab27a

JFC

Munc13-4
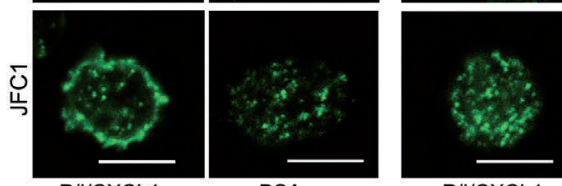

PIIICXCL1

BSA

BSA

PIICXCL1

Figure 4. Rab27a dependent vesicle transfer is impaired in Src family kinases (SFK)-knockout (ko) neutrophils, leading to an impaired basement membrane penetration. (A) Neutrophil transmigration in a transwell assay with or without CXCL1 through filters coated with Iaminin (LN), or LN, PECAM-1, and ICAM-1 (LN/P/I). All data are presented as mean \pm standard error of the mean (SEM). $* P<0.05 ; * * * P<0.001 ; n . s$. : not significant (two-way ANOVA, Sidak multiple comparison test). N=3 wildtype and 3 SFK-ko x Lyz2GFP mice, transwell assays were performed in duplicates. (B) Maximum projections of confocal microscopic images of venules in TNF $\alpha$-stimulated cremaster muscle whole mounts from wildtype and SFK-ko mice. Basement membrane was visualized by anti-LN5 (green), neutrophils by antiMRP14 (red). Surrounding muscle tissue was visualized by bright field microscopy. White arrows point at extravasated, but still attached neutrophils in SFK-ko tissue. Scale bar: $20 \mathrm{um}$. (C) and (E) immunostaining of representative wildtype and SFK-ko neutrophils on BSA- or PECAM-1/ICAM-1/CXCL1-coated (P/I/CXCL1) coverslips for (C) VLA6 and (E) VLA3 analyzed by confocal microscopy. Scale bar: $10 \mu \mathrm{m}$. (D) and (F) Quantification of ring formation for VLA6 (D) and VLA3 (F). At least 80 cells from 3 wildtype and 3 SFK-ko mice were analyzed for each condition. (G) Immunostaining of representative wildtype and SFK-ko neutrophils on BSA- or PECAM1/ICAM-1/CXCL1-coated coverslips for Rab27a (upper panel), Munc13-4 (middle panel), and JFC1 (lower panel). Scale bar is 5um. (H) Quantification of ring formation for Rab27a, Munc13-4 and JFC1. At least 80 cells from 3 wildtype and 3 SFK-ko mice were analyzed for each condition. All data are presented as mean \pm SEM. $\star P<0.05 ; P<0.005 ; * * * P<0.001$; n.s. : not significant (two-way ANOVA, Sidak multiple comparison test). 
Impaired vesicle transport in the absence of Src family kinase in neutrophils

We and others have previously shown that mobilization of vesicles containing VLA3 and VLA6 to the plasma membrane is an important process during vascular BM penetration. ${ }^{3,4,6}$ To investigate whether SFK have a role in this process, we analyzed the SFK-dependent translocation of VLA6 and VLA3 to the plasma membrane of SFKko and wildtype neutrophils. Bone marrow derived neutrophils of SFK-ko and wildtype mice were plated on BSA or, again, on a combination of PECAM-1/ICAM-1 and CXCL1 and subsequently stained for VLA6 or VLA3. Plasma membrane translocation was visualized by confocal microscopy as "ring formation" (Figure 4C and E). We observed fewer SFK-ko cells (37\%) displaying a ring of VLA6, when plated on PECAM-1/ICAM-1/CXCL1 compared to wildtype (55\%) (Figure 4D). This observation was also true for VLA3, where only $52 \%$ of SFK-deficient neutrophils showed vesicle translocation, compared to $82 \%$ of wildtype cells (Figure 4F). To test whether ring formation can also be observed in vivo, whole mount cremaster muscle stainings were conducted for VLA6, which revealed a similar trend as in vitro. In wildtype whole mounts, extravasated neutrophils displayed a ring-like staining for VLA6, while intravascular neutrophils showed only a diffuse signal. In SFK-ko whole mounts, intravascular and extravasated cells showed no clear peripheral staining (Online Supplementary Figure S2C). Next, we plated wildtype neutrophils on BSA or PECAM-1/ICAM1/CXCL1 and stained for SFK. Confocal micrographs revealed diffuse intracellular SFK staining when cells were plated on BSA (Online Supplementary Figure S2D). Upon stimulation with PECAM-1/ICAM-1/CXCL1, we observed a clear SFK signal at the cell border. Again, we quantified SFK distribution and observed a pronounced SFK translocation to the cell periphery when neutrophils were plated on PECAM-1/ICAM-1/CXCL1 (Online Supplementary Figure S2E). These experiments support a critical role for SFK by strongly interfering in vesicle trafficking of neutrophils in vitro and in vivo.

\section{Src family kinase regulate Rab27a-dependent vesicle translocation}

Vesicle trafficking in neutrophils is mainly regulated by Rab27a GTPases and their corresponding effector proteins. In neutrophils, Rab27a works in collaboration with two of its known effectors, JFC1 and Munc13-4, on vesicle trafficking and exocytosis of secretory vesicles and azurophilic granules. ${ }^{8}$ We analyzed the translocation of Rab27a, JFC1 and Munc13-4 to the cell periphery upon PECAM-1/ICAM-1/CXCL1 stimulation as described above. In wildtype neutrophils, stimulation resulted in translocation of Rab27a and its effectors (Figure 4G). While $60 \%$ of wildtype neutrophils displayed a ring-like structure, only $40.1 \%$ showed Rab27a translocation in SFK-ko neutrophils (Figure 4H). Similarly, staining for JFC1 (50.1\% vs. $29.2 \%)$ and Munc13-4 (60.9\% vs. $31.3 \%)$ revealed an equal trend for wildtype vs. SFK-ko neutrophils, respectively. Taken together, these experiments show a clear SFK-dependent activation of the secretory machinery in neutrophils.

\section{NE-dependent laminin degradation is defective in Src family kinase-ko neutrophills \\ Neutrophil extravasation is not only dependent on the}

translocation of LN binding $\beta 1$ integrins, but also on the serine proteinase neutrophil elastase $(\mathrm{NE}){ }^{5,29} \mathrm{We}$ first analyzed the localization of NE in neutrophils plated on either BSA or PECAM-1/ICAM-1/CXCL1 as described above (Figure 5A). Similar to VLA3 and VLA6, we observed an increase in ring formation from $21.9 \%$ in BSA stimulated wildtype neutrophils to $59.1 \%$ of wildtype neutrophils stimulated on PECAM-1/ICAM-1/CXCL1 (Figure 5B). This upregulation of vesicle translocation was absent in SFK-ko neutrophils $(20.0 \%$ for BSA vs. $31.2 \%$ for PECAM1/ICAM-1/CXCL1 coating). In neutrophils, NE is stored in azurophilic granules together with a variety of other proteinases and antimicrobial proteins such as myeloperoxidase (MPO). Neutrophils release MPO after stimulation with TNFo. . $^{30,31}$ Thus, we additionally quantified MPO release in the serum of SFK-ko and wildtype mice by ELISA $2 \mathrm{~h}$ after TNF $\alpha$ stimulation. While we did not observe upregulation of MPO in the serum of SFK-ko mice $2 \mathrm{~h}$ after TNF $\alpha$ stimulation $(158.4 \mathrm{ng} / \mathrm{mL}$ before vs. 184.0 $\mathrm{ng} / \mathrm{mL}$ after TNFa stimulation) (Figure 5C), MPO levels were markedly increased in the serum of TNF $\alpha$ stimulated wildtype mice $(113.0 \mathrm{ng} / \mathrm{mL}$ before vs. $473.5 \mathrm{ng} / \mathrm{mL}$ after TNFo stimulation). This indicates that SFK are required for the release of azurophilic granule content including NE and MPO. Earlier studies revealed that NE is able to proteolytically cleave laminins. In addition, NE activates different matrix metalloproteinases (MMP) that are involved in matrix degradation. ${ }^{32-34}$ However, the relevance of these NE functions for neutrophil transmigration are still not completely understood. By applying a NE-fluorescent activatable substrate (NE680FAST) in postcapillary venules with or without TNF $\alpha$ stimulation we were able to investigate NE activity in wildtype and SFK-ko cremaster whole mounts. Compared to unstimulated controls, TNF $\alpha$ stimulation led to a robust NE activity signal in wildtype mice (Figure 5D). As expected, this activity was reduced in SFK-ko venules, indicating that there is reduced NE dependent substrate cleavage in the absence of SFK (Figure 5D).

Next, we investigated whether wildtype neutrophils are able to degrade BM constituents under in vitro conditions. To this end, we performed transwell experiments in a system that allows us to image neutrophil crawling and extravasation by spinning disc confocal microscopy. Neutrophils from wildtype and SFK-ko mice were isolated and stained with different cell trackers (CellTracker ${ }^{\mathrm{TM}}$ Red CMTPX Dye and CellTracker ${ }^{\mathrm{TM}}$ Green CMFDA Dye,) to image both neutrophil populations in one transmigration chamber. In addition, laminin was visualized with an Alexa-647 coupled antibody. We observed intensive degradation of LN by wildtype neutrophils only, which could be identified by the disappearance of fluorescently labeled LN over time (Figure 5E, Online Supplementary Figure S3A and Online Supplementary Mov3). When we used SFK-ko neutrophils only, no degradation of LN was observed (Online Supplementary Figure S3B). Interestingly we found that SFK-ko neutrophils, which were unable to digest the LN layer, migrated strongly towards wildtype neutrophils during the observation period (Figure $5 \mathrm{~F}$ and G and Online Supplementary Mov4). We quantified this association of SFK-ko neutrophils around wildtype cells in a randomization approach. Here, the interaction strength is a measure of the degree of dependence of spatial distribution between wildtype and SFK-ko neutrophils. We observed an interaction strength of 5.1 after 1,000 seconds 
A

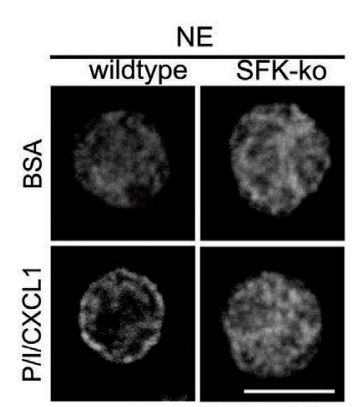

D
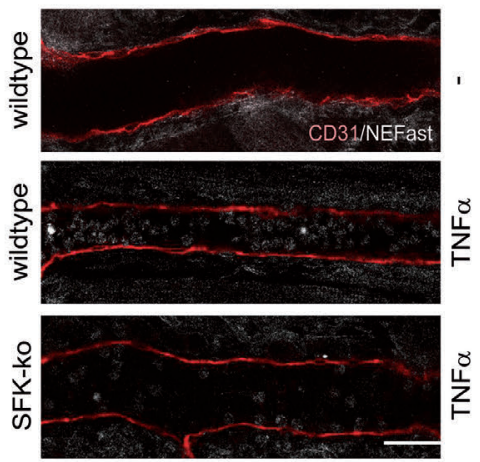

F

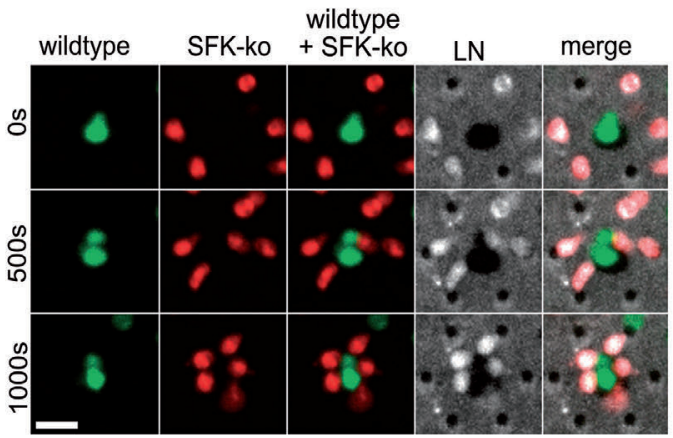

H

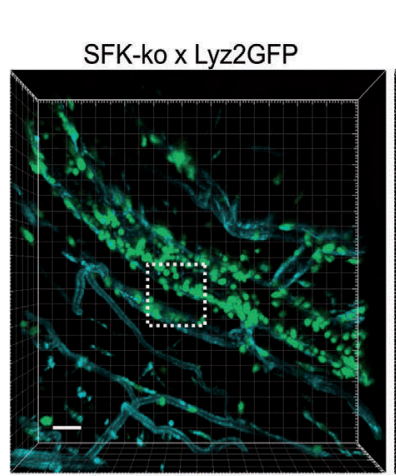

Lyz2GFP/CD31

SFK-ko x Lyz2GFP
B

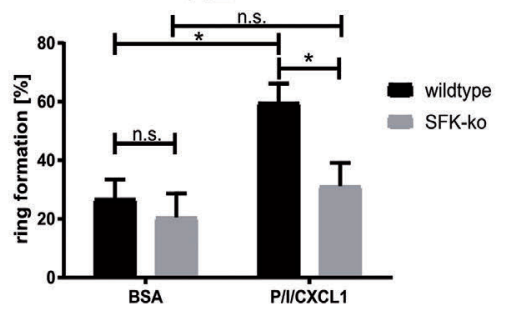

C

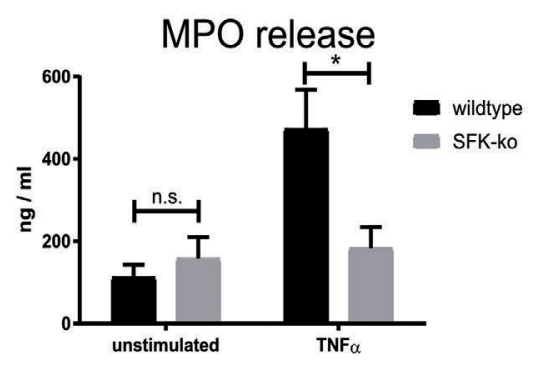

E

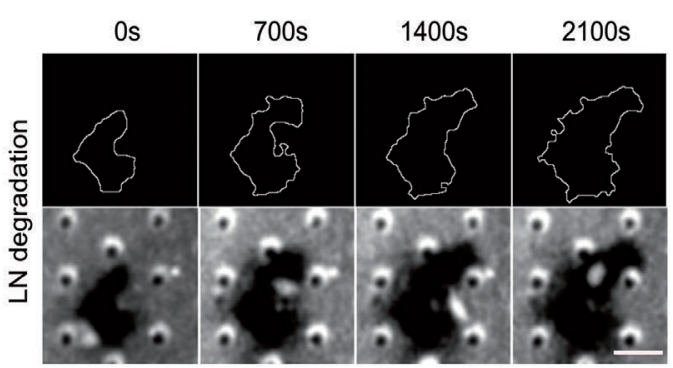

G

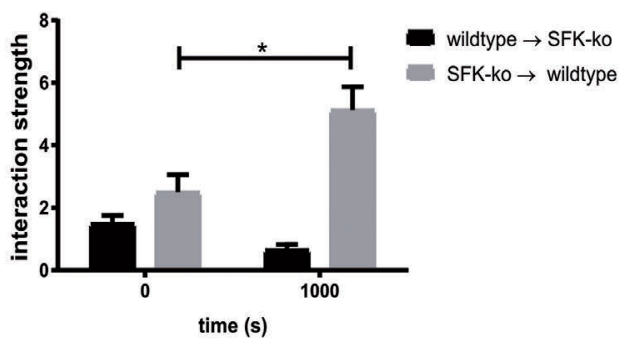

I SFK-ko x Lyz2GFP $\begin{gathered}\text { wildtype in } \\ \text { SFK-ko x Lyz2GFP }\end{gathered}$
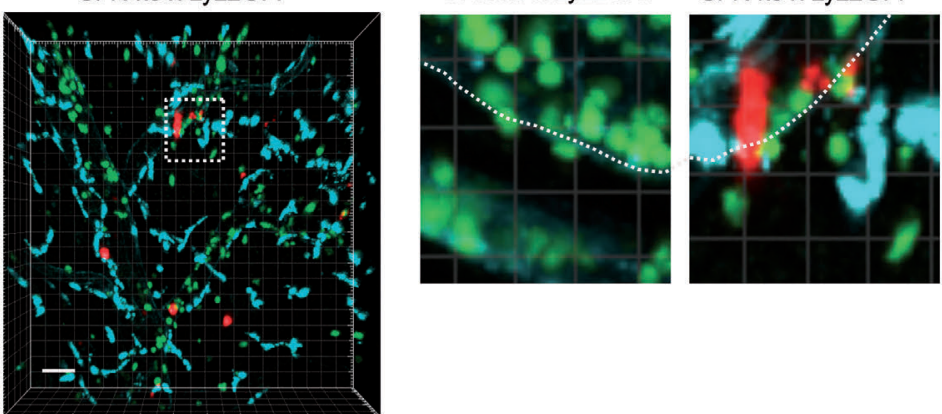

Lyz2GFP/wildtype/CD31

Figure 5. Src family kinases (SFK)-dependent neutrophil elastase (NE) translocation is critical for degradation of the basement membrane. (A) Immunostaining of representative wildtype and SFK-knockout (ko) neutrophils on BSA- or PECAM-1/ICAM-1/CXCL1-coated (P/I/CXCL1) coverslips for NE analyzed by confocal microscopy. Scale bar: $10 \mu \mathrm{m}$. N=3 wildtype and 3 SFK-ko mice. (B) Quantification of ring formation for NE. At least 80 cells from 3 wildtype and 3 SFK-ko mice were analyzed. All data are presented as mean \pm standard error of the mean (SEM). ${ }^{*} P<0.05 ; * * * P<0.001$; n.s. : not significant (two-way ANOVA, Sidak multiple comparison test). (C) Quantitative analysis for myeloidperoxidase (MPO) release in blood plasma samples from wildtype and SFK-ko mice 2 hours after TNF $\alpha$ injection by quantitative ELISA assay; 3 wildtype and 3 SFK-ko mice were analyzed. All data are presented as mean \pm SEM. $* P<0.05 ; * * P<0.005 ; * * * P<0.001 ;$ n.s. : not significant. (D) NE activity (white) within cremaster muscle venules without (upper image) or with TNF $\alpha$ middle and lower image) stimulation from wildtype and SFK-ko mice imaged by confocal microscopy. Venules were visualized using a CD31 antibody (red) ( $\mathrm{n}=3$ mice per group). Scale bar: $40 \mu \mathrm{m}$ (E) Spinning disk confocal micrographs of LN degradation (indicated by black areas compared to white LN staining) by wildtype neutrophils at indicated time points (Lower panels). White lines outline the increase in degradation area over time (Upper panels). Scale bar: $10 \mu \mathrm{m}$. (F) Spinning disk confocal micrographs of wildtype (green) and SFK-ko (red) neutrophils plated on antibody-labeled LN (white) at indicated time points. Scale bar: $10 \mu \mathrm{m}$. (G) Randomization approach to test for wildtype/SFK-ko interaction strength during migration using the nearest-neighbor analysis. (H) Multi photon microscopy of TNF $\alpha$-stimulated SFK-ko x Lyz2GFP or SFK-ko x Lyz2GFP mice with $1 \times 10^{7}$ injected deep red labeled wildtype neutrophils. Venules were visualized using a CD31 antibody (turquoise). Dotted squares indicate areas of interest (see panel I) ( $\mathrm{n=4}$ mice per group). Scale bar: $30 \mu \mathrm{m}$. (I) Close-up of vessel wall of SFK-ko $x$ Lyz2 $2^{\text {GFP }}$ or SFK-ko $x$ Lyz2 ${ }^{\text {GFP }}$ mice with $1 \times 10^{7}$ injected deep red labeled wildtype neutrophils. 
of observation (Online Supplementary Figure S3C and D), indicating that this accumulation is not random, but highly dependent. These experiments strongly support the concept of SFK-dependent BM degradation by extravasating wildtype neutrophils. In addition, our results indicate that LN degradation fragments might exert chemotactic activity.

To finally show that these in vitro findings are also relevant under in vivo conditions, we injected labeled (CellTracker ${ }^{\mathrm{TM}}$ Deep Red Dye) SFK-ko or wildtype neutrophils into Lyz2 $2^{\mathrm{GFP}}$ or SFK-ko x Lyz2 $2^{\mathrm{GFP}}$ mice, respectively, and observed neutrophil behavior using multi-photon microscopy of the mouse cremaster muscle vasculature. Compared to SFK-ko x Lyz2 ${ }^{\mathrm{GPP}}$ mice, where neutrophils accumulated in the vessel or were stuck to the abluminal side, we observed strongly elevated numbers of extravasated neutrophils in the periphery, when wildtype neutrophils were present (Figure $5 \mathrm{H}$ and Online Supplementary Mov5 and 6). Additionally, we were able to visualize SFK-ko $\mathrm{x}$ Lyz2 ${ }^{\mathrm{GFP}}$ neutrophils accumulating around adherent wildtype cells, confirming the observed behavior of SFK-ko neutrophils in the in vitro setting (Figure 5I). Also, in the experiment in which we inverted the procedure, by injecting fluorescently labeled SFK-ko neutrophils into Lyz2 $2^{\mathrm{GPP}}$ mice, we could observe extravasated SFK-ko neutrophils (Online Supplementary Figure S3E and Online Supplementary Mov7 and 8). These experiments strengthen the concept of wildtype neutrophils creating a path through the BM which enables SFK-ko neutrophils to extravasate into inflamed tissue in vivo.

\section{Discussion}

Integrin outside-in signaling is critical for neutrophil firm adhesion to and extravasation through the vessel wall into inflamed tissue. The short intracellular integrin tail has no enzymatic activity and acts exclusively as a binding site for recruited proteins, which, in turn, activate a broad range of pathways, spanning from cytoskeletal rearrangements and integrin clustering to vesicle trafficking. It has been widely accepted that tyrosine phosphorylation of ITAM motives by members of the Src family kinases is one way of integrin outside-in signaling. ${ }^{35}$ Therefore, SFK triple knockout mice ( $\mathrm{Hck}^{\wedge-} \mathrm{Fgr}^{\prime} \mathrm{Lyn} n^{\prime \prime}$, here named SFK-ko) have been used to study the function of these kinases in neutrophils in an in vivo model of acute inflammation. Interestingly, $\mathrm{Hck}^{-} \mathrm{Fgr}^{-1}$ neutrophils displayed no adhesion defect in earlier static adhesion experiments on ICAM$1,{ }^{13,36}$ while our findings show a dramatic decrease in neutrophil adhesion efficiency in inflamed cremaster muscle venules in vivo implying that SFK-dependent neutrophil adhesion is sensitive to shear stress. We also found that neutrophil extravasation in SFK-deficient mice was dramatically decreased when compared to wildtype mice, which was caused by an SFK-dependent intrinsic extravasation defect. This observation is in line with various reports in different inflammation models with SFK-ko mice $e^{14,15,21}$ where decreased numbers of neutrophils were observed. Lowell et al. linked low PMN numbers in the liver during endotoxemia to a failure of $\mathrm{Hck}^{-1} \mathrm{Fgr}^{-/}$neutrophils to rearrange their actin cytoskeleton, while cytokine production was unchanged. We clearly demonstrate that in the absence of SFK, neutrophils are unable to strongly adhere to the substrate under flow and detach in vitro and in vivo, due to defective LFA1 clustering and poor adhesion strengthening. The application of the tyrosine inhibitor Dasatinib led to a comparable shear rate dependent decrease in adherent neutrophils in inflamed venules in vivo. This strengthens previous findings of an improved outcome during sepsis after Dasatinib application, where leukocyte infiltration into the inflamed peritoneum was reduced in a dose-dependent manner. ${ }^{21}$ Interestingly, most in vitro studies were performed under steady state conditions, where integrin clustering and crawling were unaffected in SFK deficient leukocytes, ${ }^{15,37}$ neglecting the impact of shear stress on neutrophil adhesion. This phenomenon was previously observed in WASP- and mAbp1-deficient mice, where integrin clustering and migration defects only arose when neutrophils were analyzed under shear stress. ${ }^{38,39}$ Our study demonstrates that neutrophil adhesion strengthening under flow conditions is dependent on SFK which mediate clustering of integrins, the rearrangement of the actin cytoskeleton along with polarization of the cell. ${ }^{40}$ Several cytoskeleton associated proteins required for adhesion are reported phosphorylation targets of SFK (directly or indirectly via Syk) like Paxillin and Cortactin. ${ }^{41-43}$ Here we clearly show reduced phosphorylation of these adhesion-relevant proteins.

We also aimed to answer the question as to whether SFK are required for the extravasation process beyond the endothelial layer. It was suggested that leukocytes predominantly cross the vascular BM at LN low expression regions (LER), ${ }^{44}$ and recent publications, including those from our lab, showed that neutrophils need to translocate vesicles containing VLA3, VLA6 and NE to their surface in a MST1-Rab27a dependent manner in order to overcome the BM. ${ }^{3-6,45,46}$ Our results identify for the first time SFK to be an additional critical component of this process. We show that SFK-ko neutrophils fail to pass an artificial LN barrier, and even when stimulated with PECAM-1 and ICAM-1, SFK-ko neutrophils are unable to release azurophilic granules and translocate integrin containing secretory vesicles. Exocytosis and vesicle transfer in neutrophils is strongly regulated by the GTPase Rab27a and its two effectors JFC1 and Munc13-4. ${ }^{8,47}$ We demonstrate that PECAM-1/ICAM-1 and CXCL1 stimulation facilitates the translocation of all three proteins to the membrane of wildtype neutrophils, suggesting that SFK regulate vesicle transport in a Rab27a-dependent manner. How MST1 and SFK co-operate in this process is still unclear and needs further investigation. Of note, MPO release was not defective in MST1 deficient neutrophils (M Sperandio, 2019, unpublished data/personal communication) compared to SFKko neutrophils, where a marked impairment in MPO release could be observed, suggesting that SFK are involved in neutrophil vesicle trafficking independently of MST1.

The role of NE during neutrophil extravasation is still controversial, as is the role of $\mathrm{BM}$ degradation by elastases or MMP. ${ }^{5,48.52}$ However, recent findings suggest that this might, at least in part, be related to the experimental model used. Reichel et al. could clearly demonstrate decreased extravasation into inflamed cremaster muscle tissue after blockage of gelatinases by the specific inhibitor MMP-2/-9 inhibitor III. ${ }^{53}$ Furthermore, Young et al. were able to show NE-dependent extravasation using the same model..$^{29}$ It was also suggested that NE/MMP- 
dependent LN degradation creates peptides that are chemoattractant for neutrophils. ${ }^{33}$ In line with these findings, we were able to show diminished LN degradation by SFK-ko neutrophils in vitro using spinning disk confocal microscopy. Surprisingly, we observed that SFK-ko neutrophils, which are unable to degrade the LN barrier themselves, arrange around wildtype neutrophils, strongly supporting the hypothesis of the chemoattractant function of LN-fragments. Although our data seem to partly contrast with the study by Kovács et al., in which no intrinsic neutrophil migration defect was observed in the genetic absence of SFK, ${ }^{15}$ we propose that this is due to the different models used. It is also likely that the rescued extravasation into the synovial joint in their mixed chimeric mice (50\% SFK-ko, 50\% wildtype neutrophils) is in part due to restored NE or protease secretion and subsequent BM degradation by wildtype neutrophils.

Taken together, we have demonstrated a critical role for SFK in various steps along the neutrophil adhesion cascade during acute inflammation. While its effect on adhesion strengthening is the direct consequence of its prominent role directly downstream of integrin ligation and sig- naling, the regulation of vesicle trafficking during neutrophil extravasation is unexpected and provides new insights on the extravasation process through the vascular $\mathrm{BM}$, supporting the concept of neutrophil-mediated vascular BM digestion along this route.

\section{Acknowledgments}

The authors would like to thank Susanne Bierschenk and Nadine Schmidt for their excellent technical assistance, as well as Andreas Thomae and Steffen Dietzel (Core Facility Biolmaging, BioMedical Center, Ludwig-Maximilians-Universität München) for their help with confocal and multi-photon microscopy. The authors thank Thomas Graf for providing the Lyz2 $2^{\text {GFP }}$ mice.

\section{Funding}

This work was supported by grants from the European Community's Seventh Framework Programme (FP7-20072013) under grant agreement HEALTH-F4-2011-282095 (TARKINAID to MS and AM), Deutsche Forschungsgemeinschaft (SFB 914, project BO1 [MS], Z03 [MS]) and the Else Kröner-Fresenius-Stiftung (grant 2015_A68 $[I R])$.

\section{References}

1. Ley K, Laudanna C, Cybulsky MI, Nourshargh S. Getting to the site of inflammation: the leukocyte adhesion cascade updated. Nat Rev Immunol. 2007;7(9):678689

2. Sperandio M, Gleissner CA, Ley $\mathrm{K}$. Glycosylation in immune cell trafficking. Immunol Rev. 2009;230(1):97-113.

3. Hyun Y-M, Sumagin R, Sarangi PP, et al. Uropod elongation is a common final step in leukocyte extravasation through inflamed vessels. J Exp Med. 2012;209(7):1349-1362.

4. Dangerfield JP, Wang S, Nourshargh S. Blockade of $\alpha 6$ integrin inhibits IL-1- $\beta$ but not TNF- $\alpha$-induced neutrophil transmigration in vivo. J Leukoc Biol. 2005;77:159-165.

5. Wang S, Dangerfield JP, Young RE, Nourshargh S. PECAM-1, alpha6 integrins and neutrophil elastase cooperate in mediating neutrophil transmigration. J Cell Sci. 2005;118(Pt 9):2067-2076.

6. Kurz ARM, Pruenster M, Rohwedder I, et al. MST1-dependent vesicle trafficking regulates neutrophil transmigration through the vascular basement membrane. J Clin Invest. 2016;126(11):4125-4139.

7. Catz SD. The role of Rab27a in the regulation of neutrophil function. Cell Microbiol. 2014;16(9):1301-1310.

8. Brzezinska AA, Johnson JL, Munafo DB, et al. The Rab27a effectors JFC1/Slp1 and munc13-4 regulate exocytosis of neutrophil granules. Traffic. 2008;9(12):2151-2164.

9. Thomas SM, Brugge JS. Cellular Functions Regulated By Src Family Kinases. Annu Rev Cell Dev Biol. 1997;13(1):513-609.

10. Lowell CA. Inhibitory Pathways in Innate Immune Cells : Signaling Cross Talk. Cold Spring Harb Perspect Biol. 2011;1-16.

11. Futosi K, Mócsai A. Tyrosine kinase signaling pathways in neutrophils. Immunol Rev. 2016;273(1):121-139.

12. Zarbock A, Abram CL, Hundt M, Altman A, Lowell CA, Ley K. PSGL-1 engagement by E-selectin signals through Src kinase Fgr and
ITAM adapters DAP12 and FcR gamma to induce slow leukocyte rolling. J Exp Med. 2008;205(10):2339-2347.

13. Lowell CA, Fumagalli L, Berton G. Deficiency of src family kinases p59/61hck and p58c-fgr results in defective adhesiondependent neutrophil functions. J Cell Biol. 1996;133(4):895-910

14. Lowell CA, Berton G. Resistance to endotoxic shock and reduced neutrophil migration in mice deficient for the Src-family kinases Hck and Fgr. Proc Natl Acad Sci U S A. 1998;95 (13): $7580-7584$

15. Kovács M, Németh $T$, Jakus Z, et al. The Src family kinases Hck, Fgr, and Lyn are critical for the generation of the in vivo inflammatory environment without a direct role in leukocyte recruitment. J Exp Med. 2014; 211(10): 1993-2011

16. Faust N, Varas F, Kelly LM, Heck S, Graf T. Insertion of enhanced green fluorescent protein into the lysozyme gene creates mice with green fluorescent granulocytes and macrophages. Blood. 2000;96(2):719-726.

17. Lowell CA, Soriano P, Varmus HE Functional overlap in the src gene family: Inactivation of hck and fgr impairs natural immunity. Genes Dev. 1994:8(4):387-398.

18. Chan VWF, Meng F, Soriano P, DeFranco AL, Lowell CA. Characterization of the B lymphocyte populations in lyn-deficient mice and the role of lyn in signal initiation and down-regulation. Immunity. 1997;7(1):6981.

19. Pruenster M, Kurz ARM, Chung K-J, et al. Extracellular MRP8/14 is a regulator of $\beta 2$ integrin-dependent neutrophil slow rolling and adhesion. Nat Commun. 2015;6:6915.

20. Ley K, Zarbock A. Hold on to Your Endothelium: Postarrest Steps of the Leukocyte Adhesion Cascade. Immunity. 2006;25(2):185-187

21. Gonçalves-de-Albuquerque CF, Rohwedder I, Silva AR, et al. The Yin and Yang of tyrosine kinase inhibition during experimental polymicrobial sepsis. Front Immunol. 2018; 9:901.

22. Abram CL, Lowell CA. The ins and outs of leukocyte integrin signaling. Annu Rev Immunol. 2011;27339-27362

23. Yamada KM GB. Molecular interactions in cell adhesion complexes. Curr Opin Cell Biol. 1997;9(1):76-85

24. Fernandez R, Suchard SJ. Syk activation is required for spreading and $\mathrm{H} 2 \mathrm{O} 2$ release in adherent human neutrophils. J Immunol 1998;160(10):5154-5162.

25. Gallet C, Rosa JP, Habib A, Lebret M, LévyTolédano S, Maclouf J. Tyrosine phosphorylation of cortactin associated with Syk accompanies thromboxane analogueinduced platelet shape change. J Biol Chem. 1999;274(33):23610-23616.

26. Deakin NO, Turner CE. Paxillin comes of age. J Cell Sci. 2008;121(15):2435-2444.

27. Fuortes $M$, Jin WW, Nathan C. $\beta 2$ Integrindependent tyrosine phosphorylation of paxillin in human neutrophils treated with tumor necrosis factor. J Cell Biol. 1994 127(5):1477-1483

28. Wang S, Voisin M-B, Larbi KY, et al. Venular basement membranes contain specific matrix protein low expression regions that act as exit points for emigrating neutrophils. J Exp Med. 2006;203(6):1519-1532.

29. Young RE, Voisin MB, Wang S, Dangerfield J, Nourshargh S. Role of neutrophil elastase in LTB 4-induced neutrophil transmigration in vivo assessed with a specific inhibitor and neutrophil elastase deficient mice. Br J Pharmacol. 2007;151(5):628-637.

30. Burt HM, Jackson JK. The priming action of tumour necrosis factor-alpha (TNF- $\alpha$ ) and granulocyte-macrophage colony-stimulating factor (GM-CSF) on neutrophils activated by inflammatory microcrystals. Clin Exp Immunol. 1997;108(3):432-437.

31. Brandt E, Petersen F, Flad HD. Recombinant tumor necrosis factor-alpha potentiates neutrophil degranulation in response to host defense cytokines neutrophil-activating peptide 2 and IL- 8 by modulating intracellular cyclic AMP levels. J Immunol. 1992; 149(4):1356-1364

32. Okada $Y$ and Nakanishi I. Activation of matrix metallorproteinase 3 (stromelysin) 
and matrix metalloproteinase 2 (gelatinase) by human neutrophil elastase and cathepsin G. FEBS Lett. 1989;249(2):353-356.

33. Mydel P, Shipley JM, Adair-Kirk TL, et al. Neutrophil elastase cleaves laminin-332 (laminin-5) generating peptides that are chemotactic for neutrophils. J Biol Chem. 2008;283(15):9513-9522.

34. Ferry G, Lonchampt M, Pennel L, De Nanteuil G, Canet E, Tucker GC. Activation of MMP-9 by neutrophil elastase in an in vivo model of acute lung injury. FEBS Lett. 1997;402(2-3):111-115

35. Zarbock A, Lowell CA, Ley K. Syk signaling is necessary for E-selectin-induced LFA-1ICAM-1 association and rolling but not arrest. Immunity. 2007;26(6):773-783.

36. Mócsai A, Ligeti E, Lowell CA, Berton G. Adhesion-dependent degranulation of neutrophils requires the Src family kinases Fgr and Hck. J Immunol. 1999;162(2):1120-1126.

37. Giagulli C, Ottoboni L, Caveggion E, et al. The Src family Kinases Hck and Fgr are dispensable for inside-out, chemoattractantinduced signaling Regulating $\beta 2$ Integrin affinity and valency in neutrophils, but are required for $\beta 2$ integrin-mediated outside-in signaling involved in sustained adhesion. J Immunol. 2006;177(1):604-611.

38. Zhang H, Schaff UY, Green CE, et al. Impaired integrin-dependent Function in Wiskott-Aldrich syndrome protein-deficient murine and human neutrophils. Immunity. 2006;25(2):285-295.

39. Schymeinsky J, Gerstl R, Mannigel I, et al. A fundamental role of $\mathrm{mAbp} 1$ in neutrophils : impact on $\beta 2$ integrin - mediated phagocytosis and adhesion in vivo. Blood. 2009;
114(19):4209-4221.

40. Begandt D, Thome S, Sperandio M, Walzog B. How neutrophils resist shear stress at blood vessel walls: molecular mechanisms, subcellular structures, and cell-cell interactions. J Leukoc Biol. 2017;102(3):699-709.

41. Suen PW, Ilic D, Caveggion E, Berton G, Damsky CH, Lowell CA. Impaired integrinmediated signal transduction, altered cytoskeletal structure and reduced motility in Hck/Fgr deficient macrophages. J Cell Sci. 1999;112:4067-4078.

42. Mócsai A, Zhou M, Meng F, Tybulewicz VL Lowell CA. Syk is required for integrin signaling in neutrophils. Immunity. 2002; 16(4):547-558.

43. Fumagalli L, Zhang H, Baruzzi A, Lowell CA, Berton G. The Src family kinases Hck and Fgr regulate neutrophil responses to $\mathrm{N}$ formyl-methionyl-leucyl-phenylalanine. Immunol. 2007;178(6):3874-3885.

44. Voisin MB, Pröbstl D, Nourshargh S Venular basement membranes ubiquitously express matrix protein low-expression regions: characterization in multiple tissues and remodeling during inflammation. Am J Pathol. 2010;176(1):482-495.

45. Lerman YV, Lim K, Hyun YM, et al. Sepsis lethality via exacerbated tissue infiltration and TLR-induced cytokine production by neutrophils is integrin $\alpha 3 \beta 1$-dependent. Blood. 2014;124(24):3515-3523.

46. Dangerfield J, Larbi KY, Huang M-T, Dewar A, Nourshargh S. PECAM-1 (CD31) Homophilic interaction up-regulates $\alpha 6 \beta 1$ on transmigrated neutrophils in vivo and plays a functional role in the ability of $\alpha 6$ Integrins to mediate leukocyte migration through the perivascular basement membrane. J Exp Med. 2002;196(9):1201-1212.

47. Munafó DB, Johnson JL, Ellis BA Rutschmann S, Beutler B, Catz SD. Rab27a is a key component of the secretory machinery of azurophilic granules in granulocytes. Biochem J. 2007;402(2):229-239.

48. Young RE, Thompson RD, Larbi KY, et al Neutrophil elastase (NE)-deficient mice demonstrate a nonredundant role for NE in neutrophil migration, generation of proinflammatory mediators, and phagocytosis in response to zymosan particles in vivo. Immunol. 2004;172(7):4493-4502.

49. Sorokin L. The impact of the extracellular matrix on inflammation. Nat Rev Immunol. 2010;10(10):712-723.

50. Delacourt C, Hérigault S, Delclaux C, et al Protection against acute lung injury by intravenous or intratracheal pretreatment with EPI-HNE-4, a new potent neutrophil elastase inhibitor. Am J Respir Cell Mol Biol. 2002; 26(3):290-297

51. Hirche TO, Atkinson JJ, Bahr S, Belaaouaj A Deficiency in neutrophil elastase does not impair neutrophil recruitment to inflamed sites. Am J Respir Cell Mol Biol. 2004 30(4):576-584

52. Huber AR, Weiss SJ. Disruption of the subendothelial basement membrane during neutrophil diapedesis in an in vitro construct of a blood vessel wall. J Clin Invest. 1989; 83(4):1122-1136.

53. Reichel CA, Rehberg M, Bihari $\mathrm{P}$, et al Gelatinases mediate neutrophil recruitment in vivo: evidence for stimulus specificity and a critical role in collagen IV remodeling. Leukoc Biol. 2008;83(4):864-874. 